\title{
The Robertson-Walker Metric in a Pseudo-complex General Relativity
}

\author{
Peter O. Hess ${ }^{1}$, Leila Maghlaoui ${ }^{2}$ and Walter Greiner $^{2}$ \\ ${ }^{1}$ Instituto de Ciencias Nucleares, UNAM, Circuito Exterior, C.U., \\ A.P. 70-543, 04510 México D.F., Mexico \\ ${ }^{2}$ Frankfurt Institute for Advanced Studies, Johann Wolfgang Goethe Universität, \\ Ruth-Moufang-Str. 1, 60438 Frankfurt am Main, Germany
}

September 13, 2021

\begin{abstract}
We investigate the consequences of the pseudo-complex General Relativity within a pseudo-complexified Roberston-Walker metric. A contribution to the energy-momentum tensor arises, which corresponds to a dark energy and may change with the radius of the universe, i.e., time. Only when the Hubble function $H$ does not change in time, the solution is consistent with a constant $\Lambda$.
\end{abstract}

PACS: 02.40.ky, 98.80.-k

\section{Introduction}

In the past several paths have been proposed on how to extend the theory of General Relativity (GR). One of the first was Einstein himself [1, 2] who, in an attempt to unify electrodynamics with GR, extended GR to complex GR. For more recent articles on complex GR, you may consult [3, 4]. Others [5, 6, 7, 8, 9, 10, 11, 12, 13, 14, 15] introduced a maximal acceleration, related to a minimal length parameter in the theory, or [16, 17, 18, 19] tried to extend GR by introducing hyperbolic coordinates. 
The introduction of a maximal acceleration and the use of hypercomplex (a synonym for para-complex) coordinates is very much related to what was published in [20]. In [20] a pseudo-complex $(p c)$ extension of General Relativity (GR) was proposed. (To the article [20] we will refer from here on as (I).) The main objective was to investigate the analogue of the Schwarzschild metric and its consequences within the pseudo-complex extension of GR. Its possible experimental verification was and is of prime importance. The $p c$-GR is formulated in a different manner than in [16, 17, 18, 19]. Because pseudo-complex numbers exhibit a zero divisor basis (see section II), we were able to define two independent theories of GR in each zero-divisor component, which were later connected. The resulting length squared element is similar but not equal to [5, 6, 7, 8, 9, 10, 11, 12, 13, 14, 15. It contains an additional term proportional to the velocity times acceleration. As already said, the analogue of the Schwarzschild solution was studied within the new formalism. A strong heuristic assumption was made, requiring that the $p c$ scalar curvature $\mathcal{R}=0$. This did lead to strong corrections, which are excluded by the Parametrized-Post-Newton formalism (PPN) [21]. However, relaxing the condition gives corrections which are smaller and not contradicting the PPN formalism; details will be given in a forthcoming publication. Deviations of the redshift to the standard Schwarzschild case were calculated.

One of the main results and messages in (I) was: The pseudocomplex description, with its extended variational principle, contains no singularity, i.e. black holes don't exist. Furthermore, the new theory (I) automatically introduces dark energy, whose density depends in the analogue of the Schwarzschild case on the radial distance. In fact, the increase of the dark energy density towards smaller radial distances finally hinders (prevents) the collapse of a large mass. The increase of dark energy, as a function of the radial distance, depends sensitively on some functions $\xi_{k}$, which are very difficult to deduce. The results are in line with the model described in [22] where the dark energy is described by a scalar field coupled to gravity. It is interesting and quite helpful for understanding, to compare our results with those obtained within this model. In the model [22] the relativistic equations are solved numerically. As a result, dark energy accumulates around the central mass, reducing the radius of the event horizon. The advantage of this model [22] is that it provides a distribution of the scalar field, whose intensity should be proportional to our $\xi$ functions. In one case, the dark energy falls off very quickly with the radial 
distance. The disadvantage is the large numerical effort needed. The similarity between this procedure and our theory, however, indicates that both investigations might be useful for each other.

Here, in this contribution, we intend to show that the same effect introduces a dark energy in models of the Robertson-Walker (RW) type universes [29, 28]. Our theory also contains a minimal length scale. Its influence on solutions might be important for large mass concentrations [23]. Here, we will not discuss it but refer to a later publication. Whether our theory is realized in nature also depends on the predictions which can be made and on their experimental verification. In the present contribution our principal objective is to identify some consequences of the pseudo-complex theory, and to determine whether possible differences to Einsteins General Relativity may be observed.

In section II a short review on how to define $p c$ variables and their properties will be given. This is for those readers who are not yet accustomed to this mathematical structure. A simple presentation can also be found in (I). In section III we will formulate the $p c$ extension of the Robertson-Walker metric. It will be quite straight forward and the steps can be copied from [29] or any other book on GR may be consulted. For details, we will give mainly reference to [29]. In section IV we will solve the $p c$-RW model and in section $\mathrm{V}$ we shall discuss some consequences. Section VI summarizes the main conclusions.

\section{Pseudo-complex variables and pseudo- complex metric}

Here we give a brief resumé on pseudo-complex variables, helpful to understand various steps presented in this contribution. The formulas, presented here, can be used without going into the details. A more profound introduction to pseudo-complex variables is given in [24, 25], which may be consulted for better understanding.

The pseudo-complex variables are also known as hyperbolic [16, 17, hypercomplex [26] or para-complex [27]. We will continue to use the term pseudo-complex.

The pseudo-complex variables are defined via

$$
X=x_{1}+I x_{2}
$$


with $I^{2}=1$. This is similar to the common complex notation except for the different behavior of $I$. An alternative presentation is to introduce the operators

$$
\sigma_{ \pm}=\frac{1}{2}(1 \pm I)
$$

with

$$
\sigma_{ \pm}^{2}=\sigma_{ \pm}, \quad \sigma_{+} \sigma_{-}=0
$$

The $\sigma_{ \pm}$form a so called zero divisor basis, with the zero divisor defined in mathematical terms by $\boldsymbol{P}^{0}=\boldsymbol{P}_{+}^{0} \cup \boldsymbol{P}_{-}^{0}$, with $\boldsymbol{P}_{ \pm}^{0}=\left\{X=\lambda \sigma_{ \pm} \mid \lambda \epsilon \boldsymbol{R}\right\}$. The zero divisor generates all the differences to the complex number.

This basis is used to rewrite the pseudo-complex variables as

$$
X=X_{+} \sigma_{+}+X_{-} \sigma_{-},
$$

with

$$
\begin{aligned}
X_{ \pm} & =x_{1} \pm x_{2} \\
\text { or } & \\
x_{1} & =\frac{1}{2}\left(X_{+}+X_{-}\right) \quad, \quad x_{2}=\frac{1}{2}\left(X_{+}-X_{-}\right) .
\end{aligned}
$$

The pseudo-complex conjugate of a pseudo-complex variable is

$$
X^{*}=x_{1}-I x_{2}=X_{+} \sigma_{-}+X_{-} \sigma_{+} .
$$

The norm square of a pseudo-complex variable is given by

$$
|X|^{2}=X X^{*}=x_{1}^{2}-x_{2}^{2}=X_{+} X_{-} .
$$

This allows for the appearance of a positive, negative and null norm. Variables with a zero norm are members of the zero-divisor, i.e., they are either proportional to $\sigma_{+}$or $\sigma_{-}$.

It is very useful to carry out all calculations within the zero divisor basis, $\sigma_{ \pm}$. Here, all manipulations can be realized independently in both sectors, because $\sigma_{+} \sigma_{-}=0$. 
In each zero divisor component, differentiation and multiplication can be manipulated in the same way as with real or complex variables. For example, we have 25 .

$$
F(X)=F\left(X_{+}\right) \sigma_{+}+F\left(X_{-}\right) \sigma_{-}
$$

and a product of two functions $F(X)$ and $G(X)$ satisfies

$$
\begin{gathered}
F(X) G(X) \\
=\left(F\left(X_{+}\right) \sigma_{+}+F\left(X_{-}\right) \sigma_{-}\right)\left(G\left(X_{+}\right) \sigma_{+}+G\left(X_{-}\right) \sigma_{-}\right) \\
=F\left(X_{+}\right) G\left(X_{+}\right) \sigma_{+}+F\left(X_{-}\right) G\left(X_{-}\right) \sigma_{-}
\end{gathered}
$$

because $\sigma_{+} \sigma_{-}=0$ and $\sigma_{ \pm}^{2}=\sigma_{ \pm}$. As a further example, we have

$$
\frac{F(X)}{G(X)}=\frac{F\left(X_{+}\right)}{G\left(X_{+}\right)} \sigma_{+}+\frac{F\left(X_{-}\right)}{G\left(X_{-}\right)} \sigma_{-}
$$

This can be proved as follows:

$$
\begin{gathered}
\frac{F(X)}{G(X)}=\frac{F\left(X_{+}\right) \sigma_{+}+F\left(X_{-}\right) \sigma_{-}}{G\left(X_{+}\right) \sigma_{+}+G\left(X_{-}\right) \sigma_{-}} \\
=\frac{\left(F\left(X_{+}\right) \sigma_{+}+F\left(X_{-}\right) \sigma_{-}\right)\left(G\left(X_{+}\right) \sigma_{-}+G\left(X_{-}\right) \sigma_{+}\right)}{\left(G\left(X_{+}\right) \sigma_{+}+G\left(X_{-}\right) \sigma_{-}\right)\left(G\left(X_{+}\right) \sigma_{-}+G\left(X_{-}\right) \sigma_{+}\right)}
\end{gathered}
$$

where we have multiplied the numerator and denominator by the pseudo-complex conjugate of $G(X)$, using $\sigma_{+}^{*}=\sigma_{-}$. With $\sigma_{+} \sigma_{-}=0$ and $\sigma_{ \pm}^{2}=\sigma_{ \pm}$, the last expression can be written as

$$
\frac{\left(F\left(X_{+}\right) G\left(X_{-}\right) \sigma_{+}+F\left(X_{-}\right) G\left(X_{+}\right) \sigma_{-}\right)}{G\left(X_{+}\right) G\left(X_{-}\right)\left(\sigma_{+}+\sigma_{-}\right)}
$$

Because $\sigma_{+}+\sigma_{-}=1$, we arrive at Eq. (10).

Differentiation is defined as

$$
\frac{D F(X)}{D X}=\lim _{\Delta X \rightarrow 0} \frac{F(X+\Delta X)-F(X)}{\Delta X},
$$

where $D$ refers from here on to the pseudo-complex infinitesimal differential. 
A very important difference to the standard GR is the introduction of a modified variational principle. It states that the variation of an action has to be within the zero-divisor, i.e.

$$
\delta S \quad \epsilon \quad \mathcal{P}^{0},
$$

where $\mathcal{P}^{0}$ denotes the zero divisor given by all values which are either proportional to $\sigma_{+}\left(\lambda \sigma_{+}\right)$or to $\sigma_{-}\left(\lambda \sigma_{-}\right)$. For convenience, the latter is chosen in this contribution, as it was in (I). However, instead of $" \epsilon \mathcal{P}^{0}$ " we write here $"=\lambda \sigma_{-} "$. The number at the right hand side of (14) has zero norm and can be treated as a "generalized zero", thus representing a minimal extension of the variational principle.

In the $p c$-GR the metric is pseudo-complex, i.e., (see (I) for details)

$$
g_{\mu \nu}=g_{\mu \nu}^{+} \sigma_{+}+g_{\mu \nu}^{-} \sigma_{-} .
$$

Each component $\left(\sigma_{ \pm}\right)$can be treated independently, for many purposes, e.g., as how to define parallel displacement, Christoffel symbols, etc. Most of the steps, known from standard GR can be carried out analogously. This is the advantage of using the zero-divisor basis. It can also be shown that the four-divergence of the metric tensor is zero (see $(\mathrm{I}))$.

The connection of both components of the zero-divisor basis happens through the variation principle, mentioned above. It states that, with the convention used and $\delta S=\delta S_{+} \sigma_{+}+\delta S_{-} \sigma_{-}$, we have

$$
\delta S_{+}=0 \text { and } \delta S_{-}=\lambda .
$$

For the length element square

$$
\begin{aligned}
d \omega^{2} & =g_{\mu \nu} D X^{\mu} D X^{\nu} \\
& =g_{\mu \nu}^{+} D X_{+}^{\mu} D X_{+}^{\nu} \sigma_{+}+g_{\mu \nu}^{-} D X_{-}^{\mu} D X_{-}^{\nu} \sigma_{+} \\
& =D X_{\mu}^{+} D X_{+}^{\mu} \sigma_{+}+D X_{\mu}^{-} D X_{-}^{\mu} \sigma_{-},
\end{aligned}
$$

reality is imposed through

$$
d \omega^{* 2}=d \omega^{2} .
$$


This implies that $d \omega^{2}$ is equal to its pseudo real part

$$
\begin{aligned}
d \omega^{2} & =\frac{1}{2}\left(D X_{\mu}^{+} D X_{+}^{\mu}+D X_{\mu}^{-} D X_{-}^{\mu}\right) \\
& =d x_{\mu} d x^{\mu}+l^{2} d u_{\mu} d u^{\mu}
\end{aligned}
$$

where in the last step we simply substituted $X_{\mu}^{ \pm}$and $X_{ \pm}^{\mu}$ by their expressions in terms of the coordinates and four-velocities. The pseudoimaginary component has to vanish, i.e.,

$$
\begin{aligned}
0 & =\frac{1}{2}\left(D X_{\mu}^{+} D X_{+}^{\mu}-D X_{\mu}^{-} D X_{-}^{\mu}\right) \\
& =l\left(d x_{\mu} d u^{\mu}+d u_{\mu} d x^{\mu}\right) .
\end{aligned}
$$

One way to proceed is to take (19) as the final length element and impose (20) as a constriction, noting that (20) gives the dispersion relation: Integrating, this gives $u_{\mu} u^{\mu}=1$. This is a nice feature because in other models the dispersion relation is usually put in by hand.

Using Eq. (37) of (I), which reads in differential form

$$
\begin{aligned}
d x_{\mu} & =g_{\mu \nu}^{0} d x^{\nu}+l h_{\mu \nu} d u^{\nu} \\
l d u_{\mu} & =l g_{\mu \nu}^{0} d u^{\nu}+h_{\mu \nu} d x^{\nu}
\end{aligned}
$$

the length element (19) and the constriction (20) can be respectively written as

$$
\begin{aligned}
d \omega^{2}= & g_{\mu \nu}^{0}\left(d x^{\mu} d x^{\nu}+l^{2} d u^{\mu} d u^{\nu}\right) \\
& +l h_{\mu \nu}\left(d x^{\mu} d u^{\nu}+d u^{\mu} d x^{\nu}\right),
\end{aligned}
$$

and

$$
\begin{gathered}
h_{\mu \nu}\left(d x^{\mu} d x^{\nu}+l^{2} d u^{\mu} d u^{\nu}\right) \\
+l g_{\mu \nu}^{0}\left(d x^{\mu} d u^{\nu}+d u^{\mu} d x^{\nu}\right)=0
\end{gathered}
$$

These are the expressions reported in (I). 
Some models [3, 4], using complex coordinates, start with a real length element squared, defining it as $\frac{1}{2}\left[d \omega^{*}+d \omega\right]$. If one does the same with pseudo-complex coordinates, one misses then the simplifications involved, treating first the zero divisor components separately. In the complex version [3, 4, there is no zero divisor and, therefore, one does not have this advantage.

\section{Pseudo-complex Robertson-Walker Metric}

Previously we followed the steps in chapter 12.3 of the book of AdlerBazin-Schiffer [29]. Of course any other book on GR can be consulted. In this section we shall mainly repeat these steps, for sake of completeness, with the difference that the variables are now pseudo-complex. The reader will see that the formulation is identical to standard GR, with the difference of the appearance of additional functions due to the modified variational principle.

In order to proceed, one choses so called Gaussian coordinates, in which one uses a distinguished (absolute) time coordinate, thus the abandonment of a completely covariant treatment of the cosmological problem [29]. This is the price one has to pay to simplify the cosmological models and to describe physical reality in convenient mathematical terms. Such coordinates were first introduced by Gauss within a different context.

The pseudo-complex length element in Gaussian coordinates, before imposing reality, is given by

$$
\begin{aligned}
d \omega^{2} & =\left(d X^{0}\right)^{2}-e^{G\left(X^{0}, R\right)}\left(d R^{2}+R^{2} d \theta^{2}+R^{2} \sin ^{2} \theta d \phi^{2}\right) \\
& =\left(d X^{0}\right)^{2}-e^{G\left(X^{0}, R\right)} d \Sigma^{2}
\end{aligned}
$$

where we already used the pseudo-complex coordinates. $G$ is a function of time and the radial coordinate $R$. As will now be shown, $G$ can be written as the sum of the functions $g\left(X^{0}\right)$ and $f(R)$, the first one depending only on time and the second one only on $R$, i.e., $G\left(X^{0}, R\right)=g\left(X^{0}\right)+f(R)$. The starting point is the equivalence principle that two observers at two different points observe the same physics. The only difference may be in the scale the two observers use. 
Thus the ratio of the proper distance element at two different space points $R_{1}$ and $R_{2}$ must remain fixed in time:

$$
\frac{e^{G\left(X^{0}, R_{1}\right)}}{e^{G\left(X^{0}, R_{2}\right)}}=\text { const in time }
$$

i.e., this ratio must be independent of $X^{0}$. Therefore one must have

$$
G\left(X^{0}, R_{1}\right)=G\left(X^{0}, R_{2}\right)+F\left(R_{1}, R_{2}\right),
$$

which then yields for Eq. (25)

$$
\frac{e^{G\left(X^{0}, R_{2}\right)+F\left(R_{1}, R_{2}\right)}}{e^{G\left(X^{0}, R_{2}\right)}}=e^{F\left(R_{1}, R_{2}\right)}
$$

which is independent on time.

If we choose a fixed value for $R_{2}$ we can write

$$
G\left(X^{0}, R_{1}\right)=g\left(X^{0}\right)+f\left(R_{1}\right) .
$$

\section{Christoffel symbols:}

The equation for the geodesics is given by

$$
\delta \int\left[\left(\dot{X}^{0}\right)^{2}-e^{G}\left(\dot{R}^{2}+R^{2} \dot{\theta}^{2}+R^{2} \sin ^{2} \theta \dot{\phi}^{2}\right)\right] d s \quad \epsilon \quad \boldsymbol{P}^{0},
$$

with $s$ being a curve parameter. In addition, we used the new variational procedure, requiring that the variation gives a number within the zero-divisor basis.

After variation, the following equations of motion are obtained (a dot refers to the derivation with respect to $s$, the curve parameter, and a prime indicates for the function $g$ a derivative with respect to $X^{0}$ while for $f$ it is a derivative with respect to $R$ ). 


$$
\begin{array}{cl}
\ddot{X}^{0}+\frac{1}{2} g^{\prime} e^{G}\left(\dot{R}^{2}+R^{2} \dot{\theta}^{2}+R^{2} \sin ^{2} \theta \dot{\phi}^{2}\right) & =\xi_{0} \sigma_{-} \\
\ddot{R}+\frac{1}{2} f^{\prime} \dot{R}^{2}+g^{\prime} \dot{X}^{0} \dot{R} & \\
-\left(\frac{1}{2} f^{\prime}+\frac{1}{R}\right)\left(R^{2} \dot{\theta}^{2}+R^{2} \sin ^{2} \theta \dot{\phi}^{2}\right) & =\xi_{R} \sigma_{-} \\
\ddot{\theta}+2\left(\frac{1}{2} f^{\prime}+\frac{1}{R}\right) \dot{R} \dot{\theta}+g^{\prime} \dot{X}^{0} \dot{\theta}-\sin \theta \cos \theta \dot{\phi}^{2} & =\xi_{\theta} \sigma_{-} \\
\ddot{\phi}+2\left(\frac{1}{2} f^{\prime}+\frac{1}{R}\right) \dot{R} \dot{\phi}+g^{\prime} \dot{X}^{0} \dot{\phi}+2 \dot{\theta} \dot{\phi} \cot \theta & =\xi_{\phi} \sigma_{-} .
\end{array}
$$

We used the convention that on the right hand side stands an element in the zero divisor basis proportional to $\sigma_{-}$. Choosing it proportional to $\sigma_{+}$would give equivalent results, i.e., the $\sigma_{-}$and $\sigma_{+}$components are just interchanged.

Comparing this with the equation of motion

$$
\ddot{X}^{\mu}+\left\{\begin{array}{lll} 
& \mu & \\
\nu & & \lambda
\end{array}\right\} \dot{X}^{\nu} \dot{X}^{\lambda} \quad \epsilon \quad \mathcal{P}^{0},
$$

yields the non-zero Christoffel symbols (others can be deduced using the symmetry properties of the Christoffel symbols):

$$
\begin{aligned}
& \left\{\begin{array}{lll} 
& 0 & \\
1 & & 1
\end{array}\right\}=\frac{1}{2} g^{\prime} e^{G} \\
& \left\{\begin{array}{lll} 
& 0 & \\
2 & & 2
\end{array}\right\}=\frac{1}{2} g^{\prime} e^{G} R^{2} \\
& \left\{\begin{array}{lll} 
& 0 & \\
3 & & 3
\end{array}\right\}=\frac{1}{2} g^{\prime} e^{G} R^{2} \sin ^{2} \theta \\
& \left\{\begin{array}{lll} 
& 1 & \\
0 & & 1
\end{array}\right\}=\frac{1}{2} g^{\prime} \\
& \left\{\begin{array}{lll} 
& 1 & \\
1 & & 1
\end{array}\right\}=\frac{1}{2} f^{\prime} \\
& \left\{\begin{array}{lll} 
& 1 & \\
2 & & 2
\end{array}\right\}=-R^{2}\left(\frac{1}{2} f^{\prime}+\frac{1}{R}\right) \\
& \left\{\begin{array}{lll} 
& 1 & \\
3 & & 3
\end{array}\right\}=-R^{2}\left(\frac{1}{2} f^{\prime}+\frac{1}{R}\right) \sin ^{2} \theta
\end{aligned}
$$




$$
\begin{aligned}
& \left\{\begin{array}{lll} 
& 2 & \\
0 & & 2
\end{array}\right\}=\frac{1}{2} g^{\prime}=\left\{\begin{array}{ccc} 
& 3 & \\
0 & & 3
\end{array}\right\} \\
& \left\{\begin{array}{lll} 
& 2 & \\
1 & & 2
\end{array}\right\}=\left(\frac{1}{2} f^{\prime}+\frac{1}{R}\right)=\left\{\begin{array}{lll} 
& 3 & \\
1 & & 3
\end{array}\right\} \\
& \left\{\begin{array}{lll} 
& 2 & \\
3 & & 3
\end{array}\right\}=-\sin \theta \cos \theta \\
& \left\{\begin{array}{lll} 
& 3 & \\
2 & & 3
\end{array}\right\}=\cot \theta \text {. }
\end{aligned}
$$

¿From the line element we find for the determinant of the metric tensor

$$
\ln \sqrt{-g}=\frac{3}{2} g\left(X^{0}\right)+\frac{3}{2} f(R)+2 \ln R+\ln |\sin \theta| .
$$

Using the Christoffel symbols given in (32), one finds

$$
\begin{aligned}
& \left\{\begin{array}{lll} 
& \mu & \\
0 & & 0
\end{array}\right\}_{\mid \mu}=0 \\
& \left\{\begin{array}{lll} 
& \mu & \\
1 & & 1
\end{array}\right\}_{\mid \mu}=\frac{1}{2} e^{G}\left(g^{\prime \prime}+g^{\prime 2}\right)+\frac{1}{2} f^{\prime \prime} \\
& \left\{\begin{array}{lll}
{ }^{2} & \mu & \\
2 & & 2
\end{array}\right\}_{\mid \mu}=\left[\frac{1}{2} e^{G}\left(g^{\prime \prime}+g^{\prime 2}\right)\right. \\
& \left.-\left(\frac{1}{2} f^{\prime \prime}+\frac{1}{R} f^{\prime}+\frac{1}{R^{2}}\right)\right] R^{2} \\
& \left\{\begin{array}{lll} 
& \mu & \\
3 & & 3
\end{array}\right\}_{\mid \mu}=\left[\frac{1}{2} e^{G}\left(g^{\prime \prime}+g^{\prime 2}\right)\right. \\
& \left.-\left(\frac{1}{2} f^{\prime \prime}+\frac{1}{R} f^{\prime}\right)\right] R^{2} \sin ^{2} \theta \\
& -\cos ^{2} \theta \text {. }
\end{aligned}
$$

The following relations are also useful

$$
\begin{aligned}
& \left\{\begin{array}{lll}
{ } & \mu & \\
0 & & \nu
\end{array}\right\}\left\{\begin{array}{lll} 
& \nu & \\
0 & & \mu
\end{array}\right\}=\frac{3}{4} g^{\prime 2} \\
& \left\{\begin{array}{lll}
{ } \mu & \\
1 & & \nu
\end{array}\right\}\left\{\begin{array}{lll} 
& \nu & \\
1 & & \mu
\end{array}\right\}=\frac{1}{2} e^{G} g^{\prime 2}+\frac{3}{4} f^{\prime 2}+\frac{2}{R} f^{\prime}+\frac{2}{R^{2}}
\end{aligned}
$$




$$
\begin{aligned}
& \left\{\begin{array}{lll}
{ }_{2} & \mu & \\
& & \nu
\end{array}\right\}\left\{\begin{array}{lll} 
& \nu & \\
2 & & \mu
\end{array}\right\}=\left[\frac{1}{2} e^{G} g^{\prime 2}-\frac{1}{4} f^{\prime 2}-\frac{2}{R} f^{\prime}\right. \\
& \left.-\frac{2}{R^{2}}+\frac{1}{R^{2}} \cot ^{2} \theta\right] R^{2} \\
& \left\{\begin{array}{lll}
3_{3} & \mu & \\
& & \nu
\end{array}\right\}\left\{\begin{array}{ccc} 
& \nu & \\
3 & & \mu
\end{array}\right\}=\left[\frac{1}{2} e^{G} g^{\prime 2}-\frac{1}{2} f^{\prime 2}-\frac{2}{R} f^{\prime}\right. \\
& \left.-\frac{2}{R^{2}}+\frac{1}{R^{2}} \cot ^{2} \theta\right] \\
& \times R^{2} \sin ^{2} \theta \text {. }
\end{aligned}
$$

With this, the non-vanishing components of the Ricci tensor are (after some calculations using also the definition of the Ricci tensor in terms of the Christoffel symbols, identical to standard GR)

$$
\begin{aligned}
\mathcal{R}_{00}= & \frac{3}{2} g^{\prime \prime}+\frac{3}{4} g^{\prime 2} \\
\mathcal{R}_{11}= & f^{\prime \prime}+\frac{1}{R} f^{\prime}-e^{G}\left(\frac{1}{2} g^{\prime \prime}+\frac{3}{4} g^{\prime 2}\right) \\
\mathcal{R}_{22}= & {\left[\frac{1}{2} f^{\prime \prime}+\frac{1}{4} f^{\prime 2}+\frac{3}{2 R} f^{\prime}-e^{G}\left(\frac{1}{2} g^{\prime \prime}+\frac{3}{4} g^{\prime 2}\right)\right] R^{2} } \\
\mathcal{R}_{33}= & {\left[\frac{1}{2} f^{\prime \prime}+\frac{1}{4} f^{\prime 2}+\frac{3}{2 R} f^{\prime}-e^{G}\left(\frac{1}{2} g^{\prime \prime}+\frac{3}{4} g^{\prime 2}\right)\right] } \\
& \times R^{2} \sin ^{2} \theta .
\end{aligned}
$$

All other components are zero.

The last equations were just copied from [29].

To obtain the tensor component $\mathcal{R}_{\nu}^{\mu}$ we need the expression for the metric tensor and its inverse. We have

$$
g_{\mu \nu}=\left(\begin{array}{cccc}
1 & 0 & 0 & 0 \\
0 & -e^{G} & 0 & 0 \\
0 & 0 & -e^{G} R^{2} & 0 \\
0 & 0 & 0 & -e^{G} R^{2} \sin ^{2} \theta
\end{array}\right)
$$

and

$$
g^{\mu \nu}=\left(\begin{array}{cccc}
1 & 0 & 0 & 0 \\
0 & -e^{-G} & 0 & 0 \\
0 & 0 & -\frac{e^{-G}}{R^{2}} & 0 \\
0 & 0 & 0 & -\frac{e^{-G}}{R^{2} \sin ^{2} \theta}
\end{array}\right)
$$


With this we get $\left(\mathcal{R}_{\mu}^{\nu}=g^{\nu \rho} \mathcal{R}_{\mu \rho}\right)$

$$
\begin{aligned}
\mathcal{R}_{0}^{0}= & \frac{3}{2} g^{\prime \prime}+\frac{3}{4} g^{\prime 2} \\
\mathcal{R}_{1}^{1}= & \left(\frac{1}{2} g^{\prime \prime}+\frac{3}{4} g^{\prime 2}\right)-e^{-G}\left(f^{\prime \prime}+\frac{f^{\prime}}{R}\right) \\
\mathcal{R}_{2}^{2}= & \mathcal{R}_{3}^{3}=\left(\frac{1}{2} g^{\prime \prime}+\frac{3}{4} g^{\prime 2}\right) \\
& -e^{-G}\left(\frac{1}{2} f^{\prime \prime}+\frac{1}{4} f^{\prime 2}+\frac{3 f^{\prime}}{2 R}\right) .
\end{aligned}
$$

The Riemann curvature is then

$$
\mathcal{R}=3\left(g^{\prime \prime}+g^{\prime 2}\right)-2 e^{-G}\left(f^{\prime \prime}+\frac{f^{\prime 2}}{4}+\frac{2}{R} f^{\prime}\right)
$$

Denoting the energy momentum tensor by $T_{\nu}^{\mu}$ and exploiting the above results, the equations of motion are

$$
\begin{aligned}
-\frac{8 \pi \kappa}{c^{2}} T_{0}^{0} & =\left[e^{-G}\left(f^{\prime \prime}+\frac{f^{\prime 2}}{4}+\frac{2 f^{\prime}}{R}\right)-\frac{3}{4} g^{\prime 2}\right]+\xi_{0} \sigma_{-} \\
-\frac{8 \pi \kappa}{c^{2}} T_{1}^{1} & =\left[e^{-G}\left(\frac{f^{\prime 2}}{4}+\frac{f^{\prime}}{R}\right)-g^{\prime \prime}-\frac{3}{4} g^{\prime 2}\right]+\xi_{1} \sigma_{-} \\
-\frac{8 \pi \kappa}{c^{2}} T_{2}^{2} & =\left[e^{-G}\left(\frac{f^{\prime \prime}}{2}+\frac{f^{\prime}}{2 R}\right)-g^{\prime \prime}-\frac{3}{4} g^{\prime 2}\right]+\xi_{2} \sigma_{-} \\
-\frac{8 \pi \kappa}{c^{2}} T_{3}^{3} & =\left[e^{-G}\left(\frac{f^{\prime \prime}}{2}+\frac{f^{\prime}}{2 R}\right)-g^{\prime \prime}-\frac{3}{4} g^{\prime 2}\right]+\xi_{3} \sigma_{-} \\
-\frac{8 \pi \kappa}{c^{2}} T_{\nu}^{\mu} & =0, \mu \neq \nu .
\end{aligned}
$$

The $f^{\prime}(R)$ refers to the derivative with respect to $R$, while $g^{\prime}\left(X^{0}\right)$ refers to the derivative with respect to $X^{0}$. The $\xi_{\mu}$ functions appear due to the new variational principle. In [29] there appears instead the cosmological constant $\Lambda$. In principle we can add such a constant, too. However, one of the reasons not to do so, is that the $\xi$ functions will reproduce such an effect. Inspecting Eq. (41) one can identify $\xi_{k}$ as additional diagonal contributions to the energy-momentum tensor, 
times $\sigma_{-}$. Transferring $\xi_{0}$ in the first equation of (41) to the left hand side and factorizing $\frac{8 \pi \kappa}{c^{2}}$, we can associate to $\xi_{0}$ the energy density

$$
\rho_{\Lambda}=\frac{c^{2}}{8 \pi \kappa} \xi_{0} \sigma_{-}
$$

Later, when we project to the pseudo-real part, this will give a contribution to the pseudo-real energy density, associated to dark energy. This fact will be useful in understanding the results obtained further below.

\section{Solving the Equations of Motion}

Homogeneity of the matter distribution requires that

$$
T_{1}^{1}=T_{2}^{2}=T_{3}^{3}
$$

Due to the similarity to the $T_{k}^{k}$, the same argument can be used for the $\xi$ functions, giving

$$
\xi_{1}=\xi_{2}=\xi_{3} .
$$

The $\xi_{k}(k=1,2,3)$ may be functions of the time.

Taking an appropriate difference of the equation for $T_{2}^{2}$ with $T_{1}^{1}$, leads to the equation (subtract second equation in (41) from the third one in (41))

$$
f^{\prime \prime}-\frac{1}{2}\left(f^{\prime}\right)^{2}-\frac{f^{\prime}}{R}=0
$$

i.e, the same equation as given in [29]. The solution is also supplied (as proposed in [29] by direct insertion):

$$
e^{f}=\frac{b^{2}}{\left[1-\frac{a b}{4} R^{2}\right]^{2}}
$$

with $a$ and $b$ as constants. Finally, the length square element takes the form, after some redefinitions $\left(|a b|=\frac{1}{R_{0}^{2}}[29]\right)$ 


$$
d \omega^{2}=\left(d X^{0}\right)^{2}-e^{g\left(X^{0}\right)} \frac{1}{\left(1+\frac{k R^{2}}{4 R_{0}^{2}}\right)^{2}} d \Sigma^{2} .
$$

It is exactly of the same form as in standard GR, with the difference that the coordinates are now pseudo-complex. This is in distinction to the pseudo-complex Schwarzschild metric [20], where the differences appear already in the functional form of the metric. The $k$ acquires the values $k=0, \pm 1$, while $R_{0}^{2}$ is a constant, related to $a$ and $b$ via $|a b|=1 / R_{0}^{2}$. The function $g\left(X^{0}\right)$ is yet undetermined. The $k$-values of $0, \pm 1$ can be used to model different universes. However, as we know now, the $k=0$ case is the only one which is consistent with experiment [28]. This is the case we will finally study explicitly. It may serve as an example for other studies.

We will now use the co-moving pseudo-complex coordinates [29], i.e., $\dot{X}^{0}=1$ and $\dot{X}^{1}=\dot{X}^{2}=\dot{X}^{3}=0$, where the dot refers to the derivative with respect to the eigen-time.

The energy-momentum tensor takes the form

$$
\left(T_{\nu}^{\mu}\right)=\left(\begin{array}{cccc}
\rho & & & \\
& -\frac{p}{c^{2}} & & \\
& & -\frac{p}{c^{2}} & \\
& & & -\frac{p}{c^{2}}
\end{array}\right),
$$

which is quite standard. The $\rho$ is the matter density and $p$ the pressure, assumed here to be pseudo-real, though, in general they can be pseudo-complex. We nevertheless will already restrict to pseudo-real functions, as they should be.

The relevant functions in the length element take the form (see Eq. (13.3) of [29])

$$
\begin{aligned}
e^{G\left(X^{0}, R\right)} & =\frac{\boldsymbol{R}\left(X^{0}\right)^{2}}{R_{0}^{2}\left(1+k R^{2} /\left(4 R_{0}^{2}\right)\right)^{2}} \\
e^{g\left(X^{0}\right)} & =\boldsymbol{R}(t)^{2} \\
e^{f(R)} & =\frac{1}{R_{0}^{2}\left(1+k R^{2} /\left(4 R_{0}^{2}\right)\right)^{2}}
\end{aligned}
$$

which are directly obtained from the expression of the length square element, with some redefinitions. The $\boldsymbol{R}$ should not be confused with 
the pseudo-complex radius variable $R$, nor with the Riemann curvature $\mathcal{R} . \boldsymbol{R}$ is an object with a length unit and is interpreted as the pseudo-complex radius of the universe.

From now on, let us substitute $X^{0}$ by its pseudo-real part ct. For example $\boldsymbol{R}\left(X^{0}\right)$ will be written as $\boldsymbol{R}(t)$. The derivative with respect to $X^{0}$ is converted into a derivation with respect to ct, i.e., $\frac{d \boldsymbol{R}}{d(c t)}=\frac{1}{c} \frac{d \boldsymbol{R}}{d t}$ $=\frac{\boldsymbol{R}^{\prime}}{c}$.

We part now from the above equations of motion (41). The expressions in the functions $f$ and $g$ and their derivatives can be re expressed in terms of the variable $\boldsymbol{R}$ using (49). For example $f=$ $-\ln R_{0}^{2}-2 \ln \left(1+k R^{2} /\left(4 R_{0}^{2}\right)\right)$ and $g=2 \ln \boldsymbol{R}$. This and their derivatives have to be inserted into (41). Using the symmetry conditions of homogeneity (43) and (44) and the form of the energy-momentum tensor (48), the equations of motion acquire the form (remember that $\left.\boldsymbol{R}^{\prime}=\frac{d \boldsymbol{R}}{d t}\right)$

$$
\begin{aligned}
\frac{8 \pi \kappa}{c^{2}} \rho & =-\xi_{0} \sigma_{-}+\left[\frac{3 k}{\boldsymbol{R}(t)^{2}}+\frac{3}{c^{2}} \frac{\boldsymbol{R}^{\prime}(t)^{2}}{\boldsymbol{R}(t)^{2}}\right] \\
\frac{8 \pi \kappa}{c^{2}} \frac{p}{c^{2}} & =\xi_{1} \sigma_{-}-\left[\frac{k}{\boldsymbol{R}(t)^{2}}+\frac{\boldsymbol{R}^{\prime}(t)^{2}}{c^{2} \boldsymbol{R}(t)^{2}}+\frac{2 \boldsymbol{R}^{\prime \prime}(t)}{c^{2} \boldsymbol{R}(t)}\right] .
\end{aligned}
$$

The details of intermediate steps can be directly copied from any book on General Relativity, e.g. [29]. Instead of four equations we have only two, due to the symmetry conditions (43) and (44). The prime refers now to the derivative with respect to the time $t$.

Here, we will obtain one of our results, which can be extracted without detailed knowledge of the $\xi_{k}$ functions. It will be useful in order to understand the ansatz in the relation between $\xi_{1}$ and $\xi_{0}$ further below (see Eqs. (54) and (62)): Assuming that the density $\rho$ and the pressure $p$ are pseudo-real quantities, the $\sigma_{-}$component of Eq. (50) tells us that

$$
\begin{aligned}
\xi_{0} & =\frac{3 k}{\boldsymbol{R}_{-}(t)^{2}}+\frac{3}{c^{2}} \frac{\boldsymbol{R}_{-}^{\prime}(t)^{2}}{\boldsymbol{R}_{-}(t)^{2}} \\
\xi_{1} & =\frac{k}{\boldsymbol{R}_{-}(t)^{2}}+\frac{\boldsymbol{R}_{-}^{\prime}(t)^{2}}{c^{2} \boldsymbol{R}_{-}(t)^{2}}+\frac{2 \boldsymbol{R}_{-}^{\prime \prime}(t)}{c^{2} \boldsymbol{R}_{-}(t)} .
\end{aligned}
$$

Here $\boldsymbol{R}_{-}(t)$ is the $\sigma_{-}$component of the radius of the universe. In order to get an idea what this implies for $\xi_{0}$ and $\xi_{1}$, without having 
to solve the problem, we can use the experimental result $\left(\boldsymbol{R}_{r}\right.$ is the pseudo-real component of the universe)

$$
\frac{\boldsymbol{R}_{r}^{\prime}}{\boldsymbol{R}_{r}}=\mathrm{H} \quad \text { with } \quad \mathrm{H}^{\prime}<<1,
$$

where the prime refers to the derivative with respect to time and $\mathrm{H}$ is the Hubble constant. Because $\boldsymbol{R}=\boldsymbol{R}_{r}+l \boldsymbol{R}_{I}, \boldsymbol{R}_{I}$ being the pseudoimaginary component of $\boldsymbol{R}$, and $l$ is the length parameter of the theory, which is extremely small (see (I)), we also can assume that $\boldsymbol{R} \approx \boldsymbol{R}_{r}$ and, because $R_{I}=\frac{1}{2}\left(R_{+}-R_{-}\right)$, we can set $\boldsymbol{R}_{ \pm}=\approx \boldsymbol{R}_{r}$. Using this we can approximately write, assuming a nearly constant $H=\frac{\boldsymbol{R}_{r}^{\prime}}{\boldsymbol{R}_{r}}$,

$$
\begin{aligned}
\frac{\boldsymbol{R}_{r}^{\prime \prime}}{\boldsymbol{R}_{r}} & =\frac{\boldsymbol{R}_{r}^{\prime \prime}}{\boldsymbol{R}_{r}^{\prime}} \frac{\boldsymbol{R}_{r}^{\prime}}{\boldsymbol{R}_{r}}=\left(\ln \boldsymbol{R}_{r}^{\prime}\right)^{\prime} \frac{\boldsymbol{R}_{r}^{\prime}}{\boldsymbol{R}_{r}}=\left[\ln \left(\mathrm{H} \boldsymbol{R}_{r}\right)\right]^{\prime} \frac{\boldsymbol{R}_{r}^{\prime}}{\boldsymbol{R}_{r}} \\
& =\left[\ln \mathrm{H}+\ln \boldsymbol{R}_{r}\right]^{\prime} \frac{\boldsymbol{R}_{r}^{\prime}}{\boldsymbol{R}_{r}} \\
& \approx\left(\frac{\boldsymbol{R}_{r}^{\prime}}{\boldsymbol{R}_{r}}\right)^{2}=H^{2} .
\end{aligned}
$$

With that, utilizing (51) and $k=0$, we can write the $\xi_{0}$ and $\xi_{1}$ approximately as

$$
\xi_{0} \approx \frac{3}{c^{2}} \mathrm{H}^{2} \approx \xi_{1}
$$

Further below we will see that this exactly corresponds to the case of a cosmological constant not changing with the redshift. This would be an exact result, if $\mathrm{H}$ is constant all over the history of the universe. Knowing that $\mathrm{H}$ is changing in time, implies that there must be a dependence on the radius of the universe, i.e., the redshift $z$. For that we have to solve the equation of motion exactly. As we will see further below, it is not easy to get the exact form of $\xi_{0}$ and $\xi_{1}$ but rather the use of a parametrization is appropriate.

Let us now continue to solve the pc- $R W$ model:

Taking again appropriate linear combinations of (150) (the first equation of (50) plus three times the second equation of (50) and the first equation of (50) plus the second one), we get new versions of the form 


$$
\begin{aligned}
\frac{4 \pi \kappa}{c^{2}}\left(\rho+\frac{3 p}{c^{2}}\right) & =\frac{1}{2}\left(3 \xi_{1}-\xi_{0}\right) \sigma_{-}-\frac{3 \boldsymbol{R}^{\prime \prime}}{c^{2} \boldsymbol{R}} \\
\frac{4 \pi \kappa}{c^{2}}\left(\rho+\frac{p}{c^{2}}\right) & =\frac{1}{2}\left(\xi_{1}-\xi_{0}\right) \sigma_{-}+\frac{k}{\boldsymbol{R}^{2}}+\frac{\boldsymbol{R}^{\prime 2}-\boldsymbol{R} \boldsymbol{R}^{\prime \prime}}{c^{2} \boldsymbol{R}^{2}}
\end{aligned}
$$

Using that

$$
\frac{\boldsymbol{R} \boldsymbol{R}^{\prime \prime}-\boldsymbol{R}^{\prime 2}}{c^{2} \boldsymbol{R}^{2}}=\frac{d}{d t}\left(\frac{\boldsymbol{R}^{\prime}}{c^{2} \boldsymbol{R}}\right),
$$

we arrive at the equation

$$
\frac{d}{d t}\left(\frac{\boldsymbol{R}^{\prime}}{c^{2} \boldsymbol{R}}\right)=\frac{1}{2}\left(\xi_{1}-\xi_{0}\right) \sigma_{-}+\frac{k}{\boldsymbol{R}^{2}}-\frac{4 \pi \kappa}{c^{2}}\left(\rho+\frac{p}{c^{2}}\right) .
$$

Differentiation of the first equation in (50) with respect to time gives

$$
\frac{8 \pi \kappa}{c^{2}} \frac{d \rho}{d t}=-\frac{d \xi_{0}}{d t} \sigma_{-}-\frac{6 k}{\boldsymbol{R}^{3}} \boldsymbol{R}^{\prime}+\frac{6 \boldsymbol{R}^{\prime}}{\boldsymbol{R}} \frac{d}{d t}\left(\frac{1}{c^{2}} \frac{\boldsymbol{R}^{\prime}}{\boldsymbol{R}}\right) .
$$

Substituting (57) into (58) and multiplying the result by $\frac{c^{2}}{8 \pi \kappa} \boldsymbol{R}^{3}$, yields

$$
\begin{aligned}
\boldsymbol{R}^{3} \frac{d \rho}{d t}= & \frac{c^{2}}{8 \pi \kappa}\left[-\boldsymbol{R}_{-}^{3} \frac{d \xi_{0}}{d t}+3 \boldsymbol{R}_{-}^{2} \boldsymbol{R}_{-}^{\prime}\left(\xi_{1}-\xi_{0}\right)\right] \sigma_{-} \\
& -3 \boldsymbol{R}^{2} \boldsymbol{R}^{\prime}\left(\rho+\frac{p}{c^{2}}\right)
\end{aligned}
$$

Note that $3 \boldsymbol{R}^{2} \boldsymbol{R}^{\prime}=\frac{d \boldsymbol{R}^{3}}{d t}$. Shifting the last term of this equation to the left hand side leads to

$$
\frac{d}{d t}\left(\rho \boldsymbol{R}^{3}\right)+\frac{p}{c^{2}} \frac{d \boldsymbol{R}^{3}}{d t}=\frac{c^{2}}{8 \pi \kappa}\left[\frac{d \boldsymbol{R}_{-}^{3}}{d t}\left(\xi_{1}-\xi_{0}\right)-\boldsymbol{R}_{-}^{3} \frac{d \xi_{0}}{d t}\right] \sigma_{-} .
$$


Identifying the mass within a given volume of the universe by $M=\rho V$, with $V$ as a given volume, the last equation can be written as

$$
\frac{d M}{d t}+\frac{p}{c^{2}} \frac{d V}{d t}=\frac{c^{2}}{8 \pi \kappa}\left[\frac{d V_{-}}{d t}\left(\xi_{1}-\xi_{0}\right)-V_{-} \frac{d \xi_{0}}{d t}\right] \sigma_{-} .
$$

This is a local energy balance! In order to maintain local energy conservation, we have to require that the right hand side is zero. This leaves us with the condition

$$
\frac{d \xi_{0}}{d t}=\frac{d\left(\ln R_{-}^{3}\right)}{d t}\left(\xi_{1}-\xi_{0}\right)
$$

Any solution for $\xi_{0}$ and $\xi_{1}$ has to fulfill this differential equation. The negative index of $V$ refers to the fact that the equation holds in the $\sigma_{-}$ component. Using $\xi_{1}=\xi_{0}$ leads to $\frac{d \xi_{0}}{d t}=0$, or $\xi_{0}=\xi_{1}=\Lambda=$ const. I.e., for this case we recover the model with a cosmological constant not changing with time. This equation is not sufficient to solve for $\xi_{0}$ and $\xi_{1}$; in fact one condition is missing.

The first equation in (55) has the usual interpretation when $\xi_{0}=\xi_{1}$ $=0$. Then the left hand side is the sum of two positive quantities, the density and the pressure. The right hand side of (55) is proportional to the acceleration $\boldsymbol{R}^{\prime \prime}$ of the radius of the universe, $\boldsymbol{R}$, multiplied by $(-1)$. This equation tells us that the acceleration of $\boldsymbol{R}$ has to be negative, i.e., we get a de-acceleration. In contrast, in the pseudo-complex description there is an additional term $\frac{1}{2}\left(3 \xi_{1}-\xi_{0}\right) \sigma_{-}$present, which might be positive. Transferring it to the left hand side may give in total a negative function in time, i.e., depending of the functional form of $\xi_{0}$ and $\xi_{1}$ in time, an accelerated phase may be reproduced or not.

Let us see whether we can get also acceleration, i.e., that $\boldsymbol{R}^{\prime \prime}>0$ in the pseudo-complex version of GR:

Using the left hand side of Eq. (60) (the right hand side is set to zero as argued below Eq. (61)), we obtain, after multiplying with $d t$,

$$
\boldsymbol{R}^{3} d \rho+3 \boldsymbol{R}^{2} \rho d \boldsymbol{R}+\frac{p}{c^{2}} 3 \boldsymbol{R}^{2} d \boldsymbol{R}=0 .
$$

Dividing by $3 \boldsymbol{R}^{3}$ we obtain

$$
\frac{d \rho}{3}+\left(\rho+\frac{p}{c^{2}}\right) \frac{d \boldsymbol{R}}{\boldsymbol{R}}=0
$$


Finally, dividing by $\left(\rho+\frac{p}{c^{2}}\right)$ yields

$$
\frac{d \rho}{3\left(\rho+\frac{p}{c^{2}}\right)}+\frac{d \boldsymbol{R}}{\boldsymbol{R}}=0 .
$$

Now we have to make an assumption on the equation of state! This is a delicate part and the results can change, depending on which equation of state we take. The equation of state may also depend on different time epochs. The basic assumptions are that i) the distribution of the mass in the universe can be treated as an ideal gas, dust or radiation, the mass being equally distributed (this is only approximately true). The equation of state is

$$
p=\alpha \rho,
$$

where $\rho$ is the energy density and $\alpha$ is zero for a model with dust, $\frac{2}{3}$ for a classical ideal gas and $\frac{1}{3}$ for a relativistic ideal gas (radiation).

With this, 65) can be solved with the solution

$$
\rho=\rho_{0} R^{-3\left(1+\frac{\alpha}{c^{2}}\right)},
$$

where the $\rho_{0}$ is a pseudo-complex integration constant. Its dimension is density.

This result is substituted into the first equation of (55), solving for $\boldsymbol{R}^{\prime \prime}$, yields

$$
\boldsymbol{R}^{\prime \prime}=\frac{c^{2}}{6}\left(3 \xi_{1}-\xi_{0}\right) R \sigma_{-}-\frac{4 \pi \kappa}{3}\left(1+\frac{3 \alpha}{c^{2}}\right) \rho_{0} \boldsymbol{R}^{-\left(2+\frac{3 \alpha}{c^{2}}\right)}
$$

We will also need the relation

$$
\begin{aligned}
\frac{d \ln V_{-}}{d t} & =\frac{1}{V_{-}} \frac{d V_{-}}{d t}=\frac{1}{\boldsymbol{R}_{-}^{3}} \frac{d}{d t} \boldsymbol{R}_{-}^{3}=\frac{3 \boldsymbol{R}_{-}^{\prime}}{\boldsymbol{R}_{-}} \\
& =\frac{d \ln \boldsymbol{R}_{-}^{3}}{d t} .
\end{aligned}
$$

Now we remember our former result that $\xi_{1}$ has to be approximately equal to $\xi_{0}$ (Eq. (154)). Due to this we can assume that the following relation also holds approximately: 


$$
\xi_{1}=\beta \xi_{0},
$$

where $\beta$ is an additional parameter of the theory, describing the deviation from a constant Hubble parameter $H$. In principle, one can also use a power expansion of $\xi_{1}$ in terms of $\xi_{0}$, which would only introduce more parameters. The $\beta$ will later be related to observable quantities, like the Hubble constant and the deceleration parameter. Eq. (70) gives us the missing condition, with the prize of having to introduce an additional parameter. Another possibility is to use the approximate expression of $\xi_{0}$ in terms of the ratio $\left(\boldsymbol{R}_{r}^{\prime} / \boldsymbol{R}_{r}\right)$, which gives $\xi_{0}=\left(3 / c^{2}\right) H$, and use experimental observations for $H$.

Using (62), we obtain for the differential equation for $\xi_{0}$

$$
\begin{aligned}
\frac{d \xi_{0}}{d t} & =(\beta-1) \frac{d\left(\ln \boldsymbol{R}_{-}^{3}\right)}{d t} \xi_{0} \\
& =\frac{d\left(\ln \boldsymbol{R}_{-}^{3(\beta-1)}\right)}{d t} \xi_{0},
\end{aligned}
$$

with the solution

$$
\xi_{0}=\Lambda \boldsymbol{R}_{-}^{3(\beta-1)} .
$$

This leaves us with the two, yet undetermined, parameters $\Lambda$ and $\beta$. There are many different scenarios:

i) $\beta=1$ : Then $\xi_{1}=\xi_{0}=\Lambda$ is constant.

ii) $\beta \neq 0$ : This will lead (see further below) to de-accelerated and accelerated systems, depending on the value of $\beta$. Also the acceleration as a function of the radius of the universe (which can be correlated to time of evolution) depends on $\beta$ and $\Lambda$.

The real part of (68) is obtained by $R_{r}^{\prime \prime}=\frac{1}{2}\left(R_{+}^{\prime \prime}+R_{-}^{\prime \prime}\right)$. Because the minimal length scale $l$ is extremely small, we can assume that $\boldsymbol{R}_{+} \approx \boldsymbol{R}_{-} \approx \boldsymbol{R}_{r}$. The real part of (68) is obtained by summing the $\sigma_{+}$and $\sigma_{-}$components and dividing the result by 2 . We will also assume that $\alpha_{+} \approx \alpha_{-}=\alpha$, which is reasonable because the $\alpha$ relates the pressure and the density, which are both pseudo-real. Using also (70) and (72) gives the final form of the equation of motion for the radius of the universe 


$$
\begin{aligned}
\boldsymbol{R}_{r}^{\prime \prime}= & \frac{c^{2}}{12}(3 \beta-1) \Lambda R_{r}^{3(\beta-1)+1} \\
& -\frac{4 \pi \kappa}{3}\left(1+\frac{3 \alpha}{c^{2}}\right) \rho_{0} \boldsymbol{R}_{r}^{-3\left(1+\frac{\alpha}{c^{2}}\right)+1} .
\end{aligned}
$$

We have assumed that the density is real. The first term comes from the $\xi$-functions.

\section{Consequences}

In this section we shall discuss the consequences of the important result (73).

When $\beta=1$ (cosmological constant), the sign of the first term in (73) is positive and contributes to the acceleration of the universe. The acceleration increases with the radius of the universe. For a general $\beta$, the acceleration is positive, as long as $\beta>\frac{1}{3}$, it is negative (deceleration) for $\beta<\frac{1}{3}$. For $\beta=\frac{1}{3}$ no additional acceleration nor deceleration takes place. The last term in (173) is always negative, i.e., it represents a contribution which contributes to the deceleration of the universe. The behavior of how the accelerating term behaves as a function in $\boldsymbol{R}_{r}$ is also determined by $\beta$. If the exponent of $\boldsymbol{R}_{r}$ is positive, the acceleration increases with $\boldsymbol{R}_{r}$, if $\beta>\frac{2}{3}$, while it decreases with $\boldsymbol{R}_{r}$ for $\beta<\frac{2}{3}$.

The solution (73) leaves space for a number of different possible scenarios. In order to proceed, we will make the following assumption: For simplicity, we assume as before that the parameter $\alpha$ and the density $\rho_{0}$ are pseudo-real, i.e., $\alpha_{+}=\alpha_{-}=\alpha$ and $\rho_{0+}=\rho_{0-}=\rho$. In this case the solution simplifies to

$$
\begin{aligned}
\boldsymbol{R}_{r}^{\prime \prime}= & \frac{c^{2}}{12}(3 \beta-1) \Lambda R_{r}^{3(\beta-1)+1} \\
& -\frac{4 \pi \kappa}{3}\left(1+\frac{3 \alpha}{c^{2}}\right) \rho_{0} \boldsymbol{R}_{r}^{-3\left(1+\frac{\alpha}{c^{2}}\right)+1} .
\end{aligned}
$$

With no dark energy, $\Lambda=0$, using (72), we have $\xi_{1}=\xi_{0}=0$ and this equation reduces to the one in [29].

Let us now discuss several particular values of $\beta$. For that purpose we define 


$$
\widetilde{\Lambda}=\frac{c^{2}}{16 \pi \kappa} \frac{\Lambda}{\rho_{0}}
$$

Note that according to (42) the $\Lambda$ is proportional to $\rho_{\Lambda}$, the density of the dark energy, with the same proportionality factor. Here $\rho_{0}$ is the mass density of the universe. Both densities are of the same order, implying that $\widetilde{\Lambda}$ is of the order of 1 .

We can now rewrite (74) into

$$
\begin{aligned}
\widetilde{\boldsymbol{R}}_{r}^{\prime \prime} & =\frac{\boldsymbol{R}_{r}^{\prime \prime}}{\left(\frac{4 \pi \kappa}{3}\right) \rho_{0}} \\
& =\widetilde{\Lambda}(3 \beta-1) \boldsymbol{R}_{r}^{3(\beta-1)+1}-\left(1+\frac{3 \alpha}{c^{2}}\right) \boldsymbol{R}_{r}^{-3\left(1+\frac{\alpha}{c^{2}}\right)+1}
\end{aligned}
$$

In what follows, we discuss the case of dust dominated universe, i.e., $\alpha=0$. For the case of a relativistic ideal gas $\alpha=\frac{1}{3}$, the results show the same characteristics. We will take arbitrarily different values of $\beta$, which are chosen such that we will have the case of the cosmological constant $\Lambda$, a case which will represent the solution of the big-rip-off and two new solutions. These solutions are not necessarily represented in nature, i.e., $\beta$ might have a different intermediate value as those in the examples. With this we get for

a) $\beta=1:\left(\xi_{1}=\xi_{0}=\Lambda\right)$

$$
\widetilde{\boldsymbol{R}}_{r}^{\prime \prime}=2 \widetilde{\Lambda} \boldsymbol{R}_{r}-\boldsymbol{R}_{r}^{-2}
$$

The universe is accelerated by the first contribution and decelerated by the second one. For small $\boldsymbol{R}_{r}$ the universe is decelerated. For large $\boldsymbol{R}_{r}$ the first term starts to dominate and the universe is from then on accelerated. The turning point is at

$$
\boldsymbol{R}_{r} \approx 0.79 / \widetilde{\Lambda}^{\frac{1}{3}}
$$

If we set the radius of today at $\boldsymbol{R}_{0}=1$, a common definition of scale for the present epoch, the result implies that for $\widetilde{\Lambda}=1$ acceleration did set in after the universe passed 80 percent of its radius. This case corresponds to a constant cosmological function $\Lambda$. 
b) $\beta=\frac{4}{3}$ :

$$
\widetilde{\boldsymbol{R}}_{r}^{\prime \prime}=3 \widetilde{\Lambda} \boldsymbol{R}_{r}^{2}-\boldsymbol{R}_{r}^{-2}
$$

In this case, the acceleration increases with the second power in $\boldsymbol{R}_{r}$, stronger than only with a cosmological constant. The $\xi_{0}$ function (72) is then given by $\Lambda \boldsymbol{R}_{-}$, i.e., the dark energy density, represented by $\xi_{0}$, increases with the radius of the universe.. This is like the big rip-off, which is discussed in the literature. The break-even point, i.e. when acceleration is equal to deceleration, is reached for

$$
\boldsymbol{R}_{r}=1 /(3 \widetilde{\Lambda})^{\frac{1}{4}}
$$

For $\widetilde{\Lambda}=1$ the break-even point is reached when the universe is $1 / 3$ of its present radius, thus, earlier than in case a).

c) $\beta=\frac{1}{2}$ : Remember that $\alpha=0$ (dust dominated universe)! Then, from (76) we get

$$
\widetilde{\boldsymbol{R}}_{r}^{\prime \prime}=\frac{1}{2} \widetilde{\Lambda} \boldsymbol{R}_{r}^{-\frac{1}{2}}-\boldsymbol{R}_{r}^{-2}
$$

In this situation, the dark energy behaves as (use Eq. (72) ) $\rho_{0}=\frac{\Lambda}{\boldsymbol{R}_{r}^{3 / 2}}$, i.e., the density of the dark energy decreases with the radius (time) of the universe.

This is really a new solution! The accelerating and the decelerating parts are decreasing with the size of the universe, but at a different rate. For small $\mathbf{R}_{r}$, the second term dominates and the universe is decelerated, while for sufficient large $\boldsymbol{R}_{r}$ the first, accelerating, term dominates and the universe is accelerated! The break-even point is at

$$
\boldsymbol{R}_{r} \approx 2^{\frac{2}{3}} / \widetilde{\Lambda}^{\frac{2}{3}}
$$

i.e., for $\widetilde{\Lambda}=1$ the universe at this break-even point will be at about $2^{\frac{2}{3}} \approx 1.59$ times of its present radius. Of course, this can be changed using different values of $\widetilde{\Lambda}$. For $\widetilde{\Lambda}=3$ the break-even point is at 76 percent of the radius of the universe. This case is plotted in figure 1 . 
The universe starts to be accelerated after having reached $\boldsymbol{R}_{r}=0.76$ (units in $\boldsymbol{R}_{r 0}$ ). After that the acceleration increases. However, having reached the radius $\boldsymbol{R}_{r} \approx 1.9$, i.e., nearly twice the actual radius of the universe, it reaches a maximum and after that the acceleration is decreasing, reaching asymptotically zero. This universe will never collapse but reach an asymptotically non-accelerating state

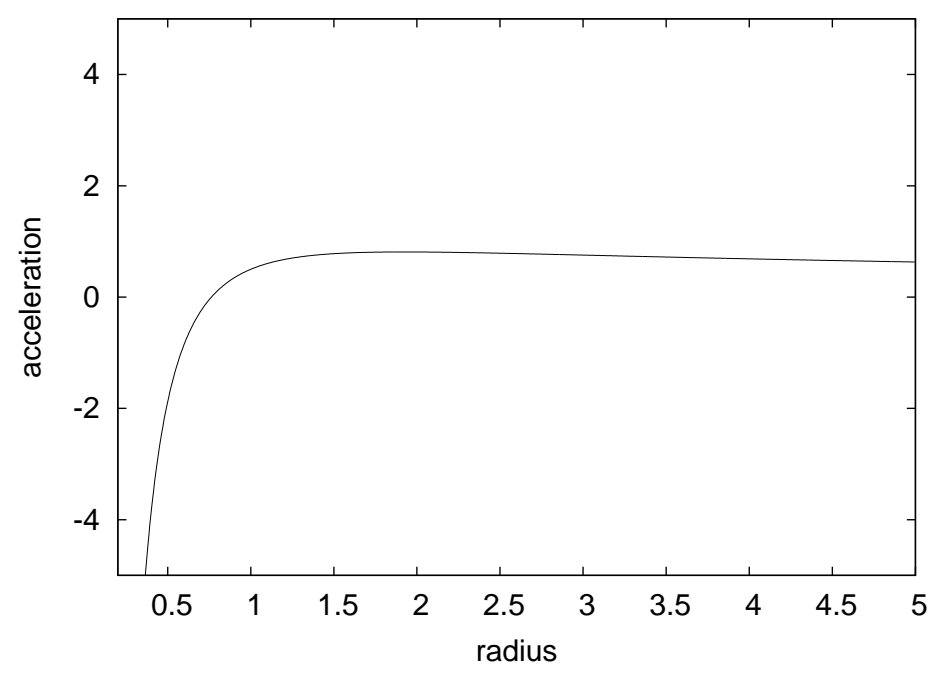

Figure 1: Dependence of the scaled acceleration as a function of $\boldsymbol{R}_{r}$, the radius of the universe, for $\beta=\frac{1}{2}$. In this figure $\widetilde{\Lambda}=3$. The maximum of this function can be deduced from (181), giving $\boldsymbol{R}_{\max }=(8 / \widetilde{\Lambda})^{\frac{2}{3}}=1.923$. The maximum can be barely seen in the figure due to the extreme slow decrease of the function. The question is also: Where are we now? Before or after the maximum?

d) $\beta=\frac{2}{3}$ : Remember that $\alpha=0$ (dust dominated universe)! Then, from (76) we get

$$
\widetilde{\boldsymbol{R}}_{r}^{\prime \prime}=\widetilde{\Lambda}-\boldsymbol{R}_{r}^{-2} .
$$

This is also a new solution. The break-even point is now at

$$
\boldsymbol{R}_{r} \approx 1 / \sqrt{\widetilde{\Lambda}}
$$


This solution is also special in the sense that the asymptotic acceleration of the universe is constant $\left(\boldsymbol{R}_{r}^{\prime \prime}=\widetilde{\Lambda}\right)$. Using (72) leads to the dependence $\rho_{0}=\Lambda / \boldsymbol{R}_{r}$ of the dark energy on the radius of the universe, i.e., it also decreases with the radius (time) of the universe.



Figure 2: Dependence of the scaled acceleration as a function in $\boldsymbol{R}_{r}$, the radius of the universe, for $\beta=\frac{2}{3}$. In this figure, the $\widetilde{\Lambda}=4$.

In all cases $\widetilde{\Lambda}$ can be fitted to the observation at which stage the net acceleration did set in, overcoming the deceleration term in (74). The new part here is that other solutions exist than the standard ones: i) There is the possibility of a constant asymptotic acceleration.

ii) In another solution, the expansion of the universe, after its decelerating period, gets accelerated. The accelerations reach a maximum and vanish asymptotically. In this case the universe approaches, for large times, an ever expanding, non-accelerating phase.

iii) In all cases, the universe is first decelerated and after a so-called break-even point it starts to accelerate.

iv) Of course, all standard solutions are obtained (cosmological constant and rip-off).

In order to calculate numerically observable consequences, we have to know the exact form of the $\xi_{k}$ functions, which we were unable to deduce from first principles. One possibility is to use the calculated distribution of dark energy, as for example done in [22]. An alternative is to use the parametrization given in (70). This implies the use 
of an additional parameter $(\beta)$ and is equivalent to known considerations in the literature [28]. The acceleration in each solution is a consequence of the $\xi_{k}$ functions. As discussed above, they represent contributions to the energy-momentum tensor, equivalent to the dark energy. In the model considered, this dark energy is in general not a constant but may vary in time, i.e., with the radius of the universe.

\section{Extraction of $\beta$ :}

We can try to connect the value of $\beta$ to observable quantities. For that, we start from Eq. (53), without the approximation in the last line. We get

$$
\begin{aligned}
\frac{\boldsymbol{R}_{r}^{\prime \prime}}{\boldsymbol{R}_{r}} & =\left[\frac{H^{\prime}}{H}+\frac{\boldsymbol{R}_{r}^{\prime}}{\boldsymbol{R}_{r}}\right] \frac{\boldsymbol{R}_{r}^{\prime}}{\boldsymbol{R}_{r}} \\
& =\left[\frac{H^{\prime}}{H}+H\right] H=H^{\prime}+H^{2} .
\end{aligned}
$$

Substituting this into the expression for $\xi_{1}$ (Eq. (51)), setting $k=0$, we obtain

$$
\begin{aligned}
\xi_{1} & =\frac{1}{c^{2}} H^{2}+\frac{2}{c^{2}}\left(H^{\prime}+H^{2}\right) \\
& =\frac{3}{c^{2}} H^{2}+\frac{2}{c^{2}} H^{\prime} \\
& =\xi_{0}+\frac{2}{c^{2}} H^{\prime} \\
& =\beta \xi_{0},
\end{aligned}
$$

where we have used that $\xi_{1}=\beta \xi_{0}$. Using $\xi_{0}=\frac{3}{c^{2}} H^{2}$ (see (54)) and solving for $\beta$, we obtain the final result

$$
\beta=1+\frac{2}{3} \frac{H^{\prime}}{H^{2}}
$$

We obtained further above that for $\beta>\frac{1}{3}$ the universe is accelerated after a given radius. This corresponds to $\frac{H^{\prime}}{H^{2}}>-1$. In order to get deeper insight, we use the deceleration parameter, which is a measure whether the universe is accelerated or decelerated, depending 
on the sign of this parameter. The deceleration parameter is defined as [29] $q=-\frac{\boldsymbol{R}_{r}^{\prime \prime} \boldsymbol{R}_{r}}{\boldsymbol{R}_{r}^{\prime 2}}$, which gives $q=-\left[1+\frac{H^{\prime}}{H^{2}}\right]$. The universe is accelerated when $\beta<\frac{1}{3}$, or $\frac{H^{\prime}}{H^{2}}<-1$, or $q>0$. The acceleration increases with $\boldsymbol{R}_{r}$ when $\beta>\frac{2}{3}$, or $\frac{H^{\prime}}{H^{2}}>-\frac{1}{2}$, or $q<-\frac{1}{2}$.

In conclusion, a measurement of the change of the Hubble constant with time will lead to a determination of the parameter $\beta$ as a function of time. Though, the last considerations clarify the role of $\beta$, we are suffering still by the problem that we have to know the solution of $H=\frac{\boldsymbol{R}_{r}^{\prime}}{\boldsymbol{R}_{r}}$. This can be done up to now only through the experimental measurement of the Hubble parameter $H$.

\section{A model including dust and radiation, $\mathrm{k}=0$ :}

Up to now, we did only consider one density component (dust or radiation) and the pseudo-complex contribution, given by the $\xi_{k}$ functions. Realistic models involve both components, as can be seen in [28]. Expressing the ratio of the radii $\boldsymbol{R}_{r 0}$ and $\boldsymbol{R}_{r}$, the present radius of the universe and the one at a redshift $z$, respectively, in terms of the redshift, we obtain [29, 28]

$$
\frac{\boldsymbol{R}_{r 0}}{\boldsymbol{R}_{r}}=(1+z)
$$

where the index $r$ refers to the real value of the radius. We obtain for the ratio of the velocity and the the radius of the universe [28]

$$
\frac{\boldsymbol{R}_{r}^{\prime}}{\boldsymbol{R}_{r}}=H=H_{0}^{2}\left\{\Omega_{d}(1+z)^{3}+\Omega_{r}(1+z)^{4}+\Omega_{\Lambda} f(z)\right\}
$$

where the index $d$ refers to the dust part and the index $r$ to the radiation part. We do not include the contribution due to $k \neq 0$, because we consider a flat universe. The factor $H_{0}^{2}$ is the square of the present Hubble constant. Using our previous result, the function $f(z)$ is given by

$$
f(z)=(1+z)^{3(\beta-1)}=(1+z)^{3(1+w)},
$$

$(\beta=2+w)$ where we made a connection to the notation used in [28]. Our result states that when the Hubble constant changes in time, 
there must be a deviation from $\beta=1$, which corresponds to $w=-1$, the case of a cosmological constant, constant in time. The deviation from $\beta=1$ cannot be large when $H^{\prime}$, the time derivative of the Hubble constant, is small. Up to now, we only find a parametrization of the $\xi_{k}$ functions in terms of the Hubble parameter or the parameter $\beta$. The deeper origin of the value of $\xi_{k}$ can probably explained by fundamental theories like string theories.

Note, that the relation $\beta=2+w$ with (87) gives a relation of $w$ to the Hubble parameter and its derivative in time, which makes definite predictions on $w$, once $H$ and $H^{\prime}$ are known. To our knowledge, this is not presented elsewhere.

\section{Conclusions}

We have applied the pseudo-complex formalism to extend the RobertsonWalker model of the universe to the $p c-$ RW model. The main results are that

1) The model introduces automatically a contribution which is equal to the cosmological constant or dark energy which may depend on the radius of the universe.

2) The cosmological "constant" is a constant when the Hubble constant is constant too. When the Hubble constant changes slightly with time, our model predicts deviations from the cosmological constant, depending on the redshift (time of expansion). The amount of deviations depends on the exact form of the $\xi_{k}$ functions.

3) The deviation can be obtained, once the radius of the universe, as a function of time, is known. Within our theory, we obtain several possible dependencies of the dark energy as a function in the radius of the universe, depending on the parameter $\beta$.

4) We also obtained several possible evolutions of the universe. Besides the solution of a constant dark energy density and the rip-off scenario, we also obtained solutions where the acceleration tends for infinite time towards zero or a constant value (see Figs. 1 and 2 ).

5) We obtained a relation between $w=\beta-2$ and $H$ and $H^{\prime}$. Once $H$ and $H^{\prime}$ are known, the $w$ value can be deduced.

The origin of the $\xi_{k}$ functions might have a deeper microscopic origin, which we do not explore here. Probably, only such a deeper microscopic understanding will fix the dependence of $\xi_{k}$ on the radius of the universe (see for example [22]). Nevertheless, the classical 
picture presented here enlightens and simplifies the description of different possible evolution scenarios of the universe.

We have not yet investigated the role of the minimal length parameter $l$, which also appears in the pseudo-complex formulation. In field theory its function is to render the theory regularized [30]. We suspect that this also happens in the pseudo-complex formulation of General Relativity and might give a hint on how to quantize this theory. In a future publication we intend to investigate the role of the minimal length scale $l$.

We saw that the modified variational principle $\delta S \epsilon \mathbf{P}^{0}$ has important consequences as the appearance of dark energy. It also provides a simpler description of effects of the dark energy, obtained via quite involved numerical calculations, as for example in [22]. These features are a hint that the variational principle has to be probably modified as proposed.

\section{Acknowledgments}

P.O.H. wants to express sincere gratitude for the possibility to work at the Frankfurt Institute of Advanced Studies and of the excellent

working atmosphere encountered there. He also acknowledges financial support from DGAPA and CONACyT.

\section{References}

[1] A. Einstein, Ann. Math. 46, 578 (1945).

[2] A. Einstein, Rev. Mod. Phys. 20. 35 (1948).

[3] C. Mantz and T. Prokopec, arXiv:gr-qc - 0804.0213v1, 2008.

[4] D. Lovelook, Annali di Matematica Pura 83, No. 1 (1969), 43.

[5] E. R. Caianiello, Nuovo Cim. Lett. 32, 65 (1981).

[6] H. E. Brandt, Found. Phys. Lett. 2, 39 (1989).

[7] H. E. Brandt, Found. Phys. Lett. 4, 523 (1989).

[8] H. E. Brandt, Found. Phys. Lett. 6, 245 (1993).

[9] R. G. Beil, Found. Phys. 33, 1107 (2003).

[10] R. G. Beil, Int. J. Theor. Phys. 26, 189 (1987).

[11] R. G. Beil, Int. J. Theor. Phys. 28, 659 (1989). 
[12] R. G. Beil, Int. J. Theor. Phys. 31, 1025 (1992).

[13] J. W. Moffat, Phys. Rev. D 19, 3554 (1979).

[14] G. Kunstatter, J. W. Moffat and J. Malzan, J. Math. Phys. 24, 886 (1983).

[15] G. Kunstatter and R. Yates, J. Phys. A 14, 847 (1981).

[16] A. Crumeyrolle, Ann. de la Fac. des Sciences de Toulouse, $4^{\mathrm{e}}$ série, 26, 105 (1962).

[17] A. Crumeyrolle, Riv. Mat. Univ. Parma (2) 5, 85 (1964).

[18] R.-L. Clerc, Ann. de L'I.H.P. Section A 12, No. 4, 343 (1970).

[19] R.-L. Clerc, Ann. de L'I.H.P. Section A 17, No. 3, 227 (1972).

[20] P. O.Hess and W. Greiner, Int. J. Mod. Phys. E 18 (2009), 51.

[21] C. W. Misner, K. S. Thorne, J. A. Wheeler, Gravitation, (W. H. Freeman Company, San Francisco, 1973)

[22] J. A. González and F. S. Guzmán, Phys. Rev. D 79 (2009), 121501.

[23] A. Feoli, G. Lambiase, G. Papini and G. Scarpetta, Phys. Lett A 263 (1999), 147.

[24] P. O. Hess and W. Greiner, JPG (2007).

[25] P. O. Hess and W. Greiner, Int. J. Mod. Phys. E (2007).

[26] I. L. Kantor, A. S. Solodovnikov, Hypercomplex Numbers. An Elementary Introduction to Algebra, (Springer, Heidelberg,1989).

[27] V. Cruceanu, P. Fortuny and P. M. Gadea, Rocky Mountain J. of Math. 26, 83 (1996).

[28] P. J. E. Peebles, Rev. Mod. Phys. 75 (2003), 559.

[29] R. Adler, M. Bazin and M. Schiffer, Introduction to General Relativity, (McGraw Hill, New York, 1975).

[30] P. O. Hess and W. Greiner, Int. J. Mod. Phys. E 16 (2007), 1643. 